\title{
Article \\ Energy Transformation on Vault in Elite Artistic Gymnastics: Comparisons between Simple and Difficult Tsukahara and Yurchenko Vaults
}

\author{
Christoph Schärer *, Micah Gross, Silvio Lorenzetti (D) and Klaus Hübner
}

Citation: Schärer, C.; Gross, M.; Lorenzetti, S.; Hübner, K. Energy Transformation on Vault in Elite Artistic Gymnastics: Comparisons between Simple and Difficult Tsukahara and Yurchenko Vaults. Appl. Sci. 2021, 11, 9484. https:// doi.org/10.3390/app11209484

Academic Editor: Alessandro de Sire

Received: 2 September 2021

Accepted: 4 October 2021

Published: 13 October 2021

Publisher's Note: MDPI stays neutral with regard to jurisdictional claims in published maps and institutional affiliations.

Copyright: (c) 2021 by the authors. Licensee MDPI, Basel, Switzerland. This article is an open access article distributed under the terms and conditions of the Creative Commons Attribution (CC BY) license (https:// creativecommons.org/licenses/by/ $4.0 /)$.
Department of Elite Sport, Swiss Federal Institute of Sport Magglingen (SFISM), 2532 Magglingen, Switzerland; micah.gross@baspo.admin.ch (M.G.); silvio.lorenzetti@baspo.admin.ch (S.L.);

klaus.huebner@baspo.admin.ch (K.H.)

* Correspondence: christoph.schaerer@baspo.admin.ch; Tel.: +41-58-467-65-04

Abstract: Performance on vault in artistic gymnastics depends on the difficulty and the execution quality of the performed vault. However, differences of kinetic energy between simple and difficult vaults remain elusive. Therefore, in this study, 48 Tsukahara and Yurchenko vaults, performed by 20 top-level gymnasts, were recorded with 3D-motion capture and the flux of translational (TKE), angular kinetic (AKE), potential (PE), and total energy were calculated and compared. Results revealed that upon initial springboard contact, almost all of the kinetic energy for Tsukahara vaults is comprised of TKE, whereas Yurchenko vaults were characterized by substantially less TKE, but far greater AKE (and similar PE). During springboard contact of Tsukahara vaults, AKE is increased $(+70 \%)$ and thereafter mostly preserved during push off from the table $(-6 \%)$. For Yurchenko vaults, AKE is preserved during springboard contact but reduced $(-30 \%)$ in exchange for PE at push off. During the second flight phase of Yurchenko vaults, total energy was $10 \%$ higher than at initial springboard contact (Tsukahara: $-1 \%$ ). For vaults of increasing difficulty, 5.9\% more AKE is needed for each additional $180^{\circ}$ of longitudinal-axis rotation. This knowledge may help coaches evaluate athletes' potential and focus training on appropriate physical and/or technical aspects of the vault performance.

Keywords: translation energy; rotation energy; dynamics; acrobatic; twisting movements; somersault

\section{Introduction}

On vault in artistic gymnastics, the final score is calculated as the sum of the difficulty score (D-score) and the execution score (E-score) [1]. Therefore, in addition to a clean execution, gymnasts must perform vaults of sufficient difficulty in order to attain a top ranking in competitions. Higher D-scores are mainly associated with changes in body position (tucked, piked, and layout) or an increased number of rotations around the longitudinal or transverse axis during the second flight phase [2]. These factors generally involve greater flight time and specifically greater translational and/or rotational energy during the second flight phase.

Although the three most common vault styles, handspring, Tsukahara (handspring with $1 / 4$ or $1 / 2$ turn in the first flight phase), and Yurchenko (round-off entry vaults (with $1 / 2$ turn) in the first flight phase) (Figure 1), have different technical and physical requirements, there is consensus that the run-up is the most important phase in order to generate the necessary energy on vault [3-7]. A high run up speed is strongly related to physical preconditions (20 m sprint speed, and explosive and reactive strength) [8] and therefore, besides training, genetics may play also a noteworthy role for the performance on vault [9].

However, this energy needs to be transformed during take-off from the springboard and push-off from the table into optimal amounts of rotational (angular momentum), potential (time of flight), and translational energy needed for the intended saltos and twists 
during the second flight phase. These amounts remain elusive. Although the vault is the most researched event of gymnastics [10], only one study reported transformations of energy (i.e., flux of energy) over the course of a vault [11]. Moreover, to the best of our knowledge, differences in energy parameters between simple and difficult vaults have never been investigated. However, such knowledge would help coaches assess the athletes' potential and to ensure their safety as they increase vault difficulty (i.e., number of rotations).
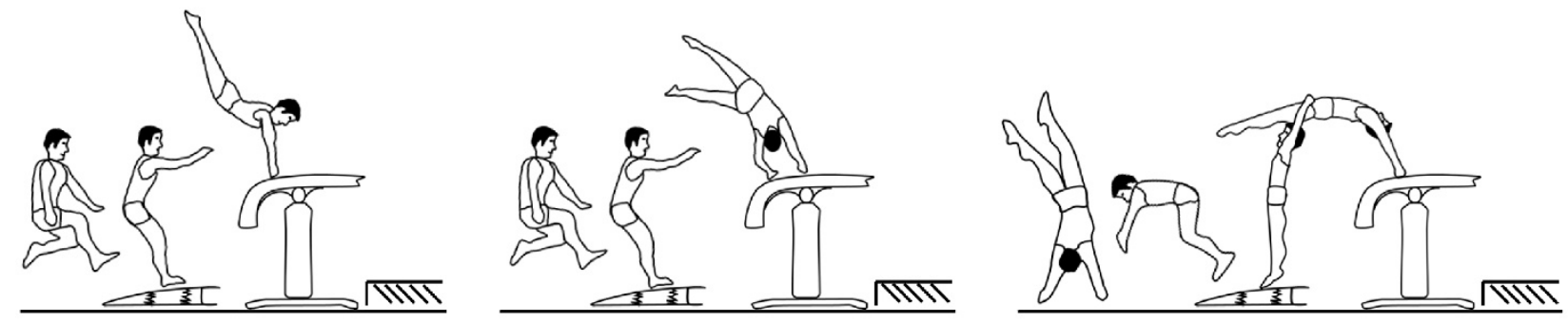

Figure 1. Different vault styles. The different first flight phases of the three most common vault styles in male artistic gymnastics. Left: Handspring; middle: Tsukahara; right: Yurchenko.

Thus, the first aim of this study was to describe and compare the flux of energy (translational, rotational, potential, and total energy) of different Tsukahara and Yurchenko style vaults. The second aim was to compare energy flux between simple and difficult Yurchenko and Tsukahara style vaults.

Therefore, we first hypothesized that for Yurchenko vaults, a substantial amount of total energy is generated with the preliminary round-off and second that the necessary amount of additional angular kinetic energy in order to perform a more difficult vault $\left(+180^{\circ}\right.$ of rotation around longitudinal axis) is (almost) negligible.

\section{Materials and Methods}

\subsection{Participants}

Twenty international and national male elite and junior gymnasts (age: $18.3 \pm 2.2 \mathrm{y}$; height: $167.6 \pm 4.9 \mathrm{~cm}$; body mass: $61.3 \pm 5.5 \mathrm{~kg}$ ) volunteered to participate. Elite athletes followed a professional training regime in the national training center, whereas junior athletes trained semi-professionally at different regional training centers. All participating athletes were members of the elite or junior national team. Prior to the measurements, athletes were informed of the benefits and risks of their participation and informed consent was obtained from all subjects involved in the study. The study was conducted according to the guidelines of the Declaration of Helsinki, and approved by the Ethics Committee of Bern, Switzerland (Project-ID: 2016-01970, 16 January 2017).

\subsection{Procedures}

After a 15-min individual warm up, each gymnast completed at least four consecutive vaults in a timespan of approximately $15 \mathrm{~min}$. Athletes had a maximum of two attempts per vault. In total, 92 vaults were performed in eight half-day sessions of data collection. For each gymnast, a maximum of four different vaults per vaulting style were analysed (only the vault with the better execution based on an E-score, judged by an expert). Included in the evaluations were 20 Yurchenko (layout: $\mathrm{n}=5 ; 360^{\circ}: \mathrm{n}=4 ; 720^{\circ}: \mathrm{n}=7 ; 900^{\circ}: \mathrm{n}=4$ ) and 28 Tsukahara (layout: $\mathrm{n}=5 ; 360^{\circ}: \mathrm{n}=11 ; 720^{\circ}: \mathrm{n}=10 ; 900^{\circ}: \mathrm{n}=2$ ) style vaults, with different numbers of rotations around longitudinal axis (Table 1). 
Table 1. Number of the different vaults performed by international (int) and national (n) elite and junior athletes in this study vaults $\left({ }^{\circ}\right.$ : degrees of rotation around longitudinal axis during the second flight phase).

\begin{tabular}{|c|c|c|c|c|c|c|c|c|c|}
\hline \multirow{2}{*}{ Athlete. } & \multirow{2}{*}{ Level } & \multicolumn{4}{|c|}{ Yurchenko Layout } & \multicolumn{4}{|c|}{ Tsukahara Layout } \\
\hline & & $0^{\circ}$ & $360^{\circ}$ & $720^{\circ}$ & $900^{\circ}$ & $\mathbf{0}^{\circ}$ & $360^{\circ}$ & $720^{\circ}$ & $900^{\circ}$ \\
\hline 1 & Junior $\mathrm{n}$ & $\mathrm{x}$ & $\mathrm{x}$ & & & & & & \\
\hline 2 & Junior $n$ & & & & & & $x$ & $\mathrm{x}$ & \\
\hline 3 & Junior int & $x$ & & $\mathrm{x}$ & $\mathrm{x}$ & $\mathrm{x}$ & $x$ & $\mathrm{x}$ & \\
\hline 4 & Junior int & & & $x$ & & & & & \\
\hline 5 & Junior n & & & & & $\mathrm{x}$ & $x$ & $\mathrm{x}$ & \\
\hline 6 & Junior $n$ & & & & & $x$ & $x$ & $x$ & \\
\hline 7 & Junior int & & & $x$ & $x$ & & & & \\
\hline 8 & Junior int & & $x$ & $x$ & & & & & \\
\hline 9 & Junior int & & & & & & $x$ & $x$ & \\
\hline 10 & Junior int & & & & & & & $x$ & \\
\hline 11 & Junior $\mathrm{n}$ & & & & & & $x$ & & \\
\hline 12 & Junior int & & & & & & $x$ & $\mathrm{x}$ & \\
\hline 13 & Junior int & & & & & $x$ & $x$ & & \\
\hline 14 & Elite int & & & & & $x$ & $x$ & $\mathrm{x}$ & \\
\hline 15 & Elite $\mathrm{n}$ & $\mathrm{x}$ & & $x$ & $x$ & & & & \\
\hline 16 & Elite $\mathrm{n}$ & & & & & & $x$ & & \\
\hline 17 & Elite int & $\mathrm{x}$ & $\mathrm{x}$ & $x$ & $x$ & & & & \\
\hline 18 & Elite int & & & & & & $x$ & $\mathrm{x}$ & $x$ \\
\hline 19 & Elite int & & & & & & & $x$ & $x$ \\
\hline 20 & Elite int & $x$ & $x$ & $x$ & & & & & \\
\hline \multicolumn{2}{|c|}{ Total } & 5 & 4 & 7 & 4 & 5 & 11 & 10 & 2 \\
\hline
\end{tabular}

All vaults were captured by 14 Vicon Vantage V5 Cameras (Vicon Motion System, Denver, CO, USA) operating at $120 \mathrm{~Hz}$. Eight cameras were placed at a height of $5.50 \mathrm{~m}$ and six were installed at $1.70 \mathrm{~m}$ (Figure 2). A measurement volume of $2 \mathrm{~m} \times 3.5 \mathrm{~m} \times 7 \mathrm{~m}$ was calibrated with a measurement error of maximally $2 \mathrm{~mm}$. Thirty-nine reflective markers were attached (with double-sided adhesive tape) to the gymnasts' body, according to Vicon Plug-in Gait full-body model (Vicon, 2010) (Figure 3). Two markers were added on gymnasts' back in order to help fill gaps in the trajectories of the original Plug-in-Gait markers. In order to calculate the Plug-in-Gait-Model, the following measurements had to be realised:

- body mass;

- height;

- length of legs (distance from superior iliac spine to lateral malleolus;

- width of ankles (distance from lateral to medial malleolus;

- $\quad$ width of knee at the level of joint gap;

- $\quad$ width of elbows (distance from lateral to medial epicondyle);

- width of wrists (distance from radial to ulnar styloid process);

- thickness of hands in the area of metacarpal bones;

- vertical distance between centre of rotation of the shoulder joint and acromion. 


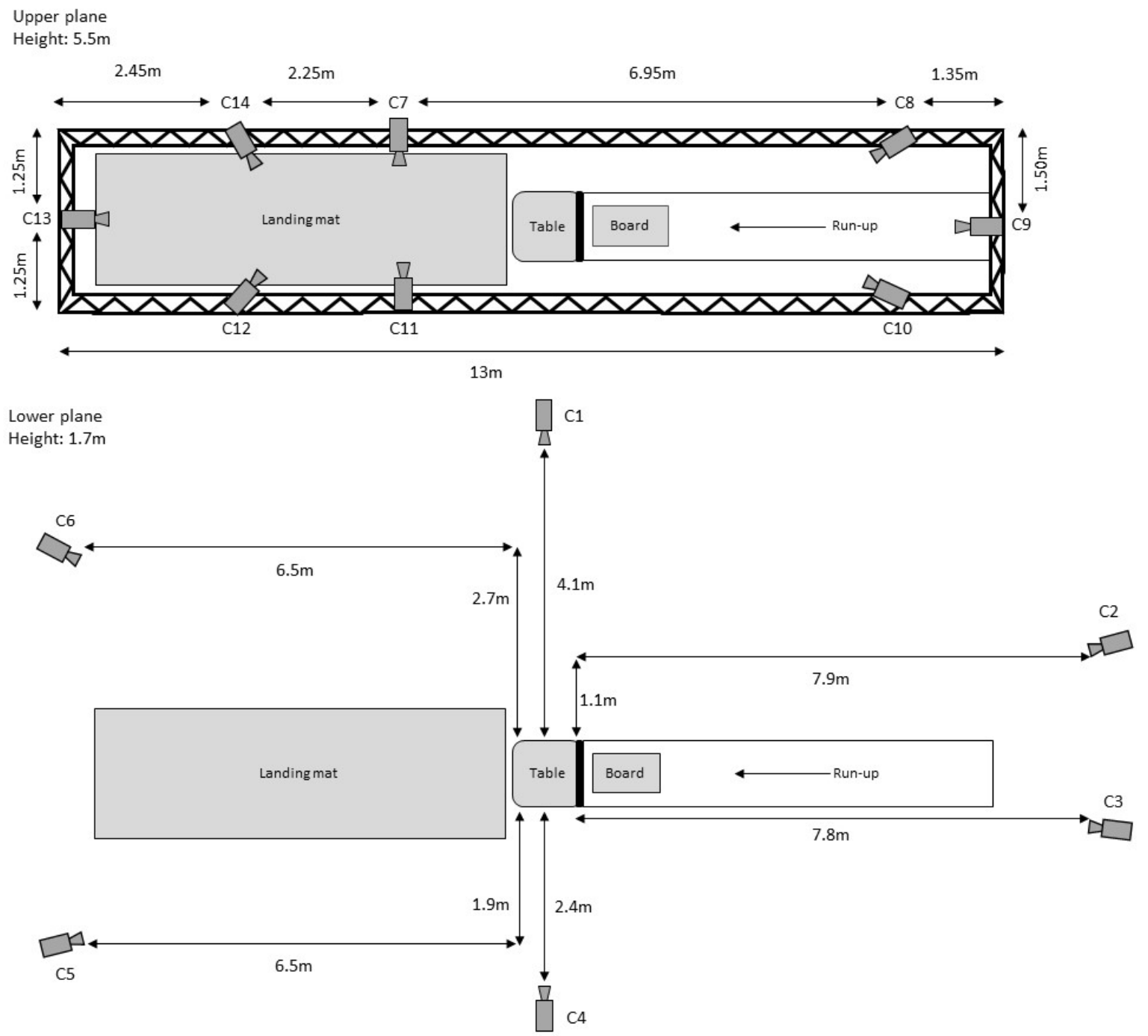

Figure 2. Arrangement of the 14 Vicon Vantage Cameras (C1 to $\mathrm{C} 14)$ for 3-dimensional motion capture of vaults.

\subsection{Energy Calculations}

Segments and Segment Properties

After labelling and filling gaps in trajectories with the built-in options (rigid body, kinematic, or pattern fill), marker trajectories were smoothed using a Woltring quanticspline routine with a mean square error (MSE) of $5 \mathrm{~mm}^{2}$. Next, the built-in Process Dynamic Plug-in Gait Model operation was executed within Nexus. Plug-in Gait processing (PIGP) creates a three-axis system for each body segment and modelled markers at joint centres and segment centres of mass, as well as a virtual marker at the body's centre of mass (CoM). As PIGP does not create a three-axis system for the body's CoM, CoM axes were created manually as a parallel-shifted copy of the thorax segment's axes.

Segment lengths were calculated as follows (italicized terms designate markers or modelled markers from the plug-in gait model):

- Head: mid forehead (mean of 'RFHD' and 'LFHD') to 'C7';

- Thorax: 'PelvisCoM' to 'C7';

- Pelvis: 'PelvisO' (origin of pelvis coordinate system, approximately the lower edge of the pelvis in the sagittal plane) to 'PelvisCoM' (approximately the upper edge of the pelvis in the sagittal plane);

- L/R Humerus: shoulder joint centre to elbow joint centre;

- L/R Radius: elbow joint centre to wrist joint centre; 
- L/R Hand: wrist joint centre to hand coordinate system origin (approximately the most distal point of the fingers);

- $\quad$ L/R Femur: hip joint centre to knee joint centre;

- L/R Tibia: knee joint centre to ankle joint centre;

- L/R Foot: ankle joint centre to foot coordinate system origin (approximately the distal end of the 5 th metatarsal).
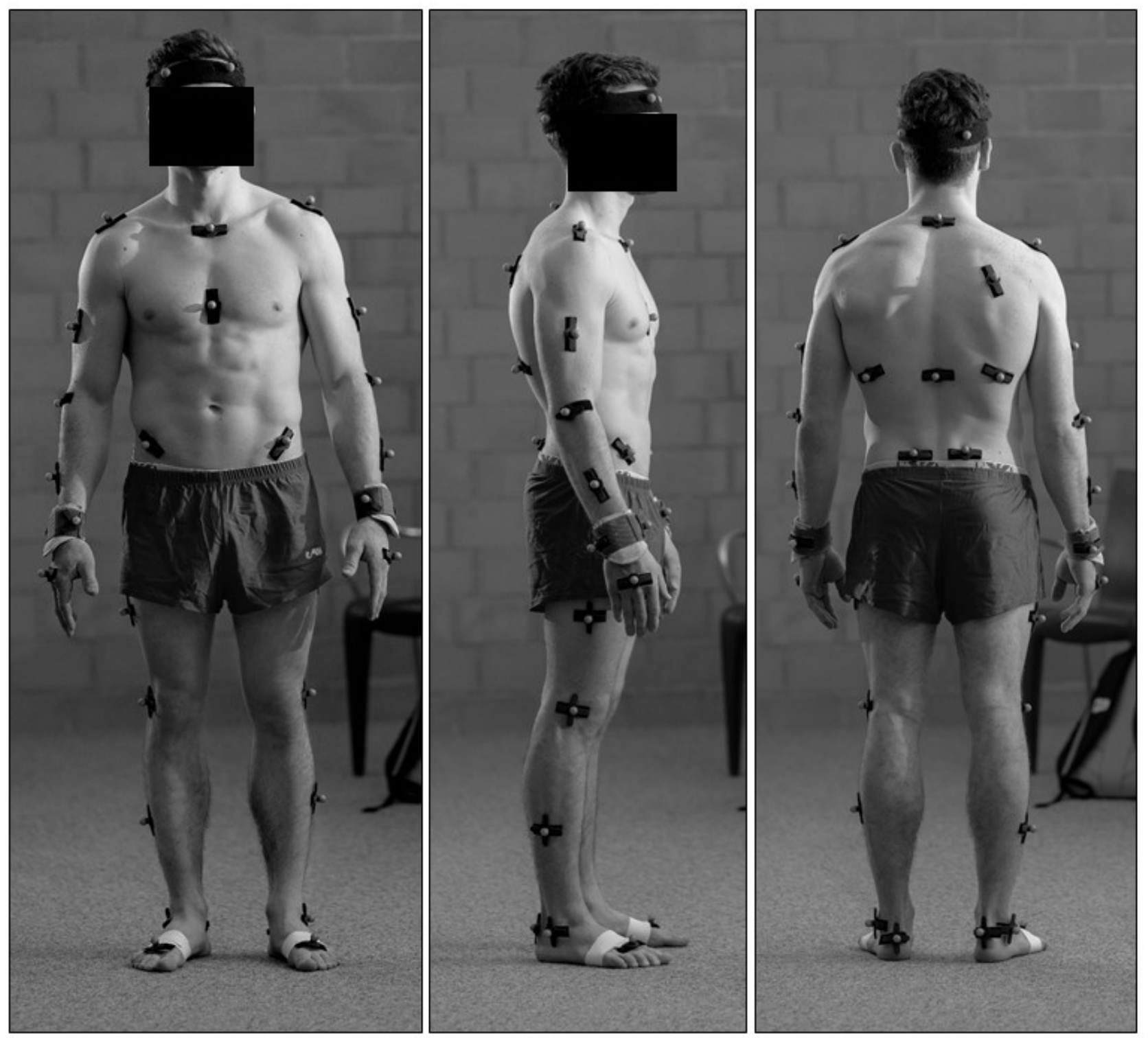

Figure 3. Positioning of the reflective markers used for 3-dimensional motion capture analysis according to the Vicon Plug-In Gate model (Vicon, 2010).

Segment lengths were calculated as described above for each frame separately. However, for further calculations, the median of all valid frames (i.e., those where PIGP was possible) was taken and assumed to be the constant for a given segment.

Segment masses and radii of gyration were determined by assuming these to be a given percent of total body mass or segment length, respectively, according to the plugin-gait reference guide [12]. Each segment was considered to be a cylinder; therefore, these radii of gyration were assumed to apply for rotation about segments' transverse and sagittal axes, whereas for rotation about its longitudinal axis, an estimate of the segment's cylindrical radius was made (Appendix A). Thereafter, using these parameters, classical 
physics, and the approaches of Kwon et al. [13], Wittenburg [14], Schüler et al. [15], and Peraire and Widnall [16], the total energy, translational kinetic energy (TKE), potential energy (PE), and angular kinetic energy (AKE) were calculated. Further details to these calculations are presented in Appendix B.

\subsection{Statistical Analysis}

Energy values for the different Tsukahara and Yurchenko vaults (layout, 360, $720^{\circ}$, and $900^{\circ}$ ) were normalized to body weight and averaged for each phase of the vault (board contact; 1st flight phase; vault contact; and 2nd flight phase). Further, energy parameters of the first two frames of each phase of each vault were averaged in order to compare simple and difficult vaults. Shapiro-Wilk tests revealed that the data were not normally distributed. Thus, first, the flux of different energy forms was averaged and analysed separately for all Tsukahara and Yurchenko vaults. Second, the differences in energy parameters between simple and difficult Tsukahara and Yurchenko style vaults (layout; $360^{\circ} ; 720^{\circ}$; and $900^{\circ}$ ) as well as differences between Tsukahara and Yurchenko vaults with the same second flight phase were calculated using Mann-Whitney-U-Tests. $p$-Values were adjusted using the Bonferroni-Holm correction [17]. Further, effect sizes were calculated $(\mathrm{r}=\mathrm{Z} / \sqrt{ } \mathrm{N})$ according to Fritz et al. [18] and rated according to Cohen [19] (small: $\geq 0.1$; medium: $\geq 0.3$; and large: $\geq 0.5$ ). The level of statistical significance was set to $p<0.05$. Statistical analysis were executed with SPSS 22 software (SPSS, Inc., Chicago, IL, USA).

\section{Results}

\subsection{Energy Transformation of Tsukahara and Yurchenko Vaults}

Upon springboard contact, considering all Tsukahara vaults, TKE comprised $72 \%$ of total energy on average (Figure 4$)$. During board contact, both total energy $(-2 \%)$ and TKE $(-26 \%)$ diminished whereas, AKE $(+70 \%)$ and PE $(+23 \%)$ increased. During table contact, total energy $(-6 \%)$, TKE $(-58 \%)$, and AKE $(-6 \%)$ decreased, but PE increased (+38\%). After push-off from the vault (during the second flight phase), total energy continued to increase by $9 \%$, achieving a maximal value during the second flight phase within $1 \%$ of that at the initial springboard contact. This increase in total energy corresponded with 33\% and $13 \%$ increases in AKE and PE, respectively.

Upon springboard contact, considering all Yurchenko vaults, average total energy consisted of 49\% TKE, 27\% PE, and 25\% AKE (Figure 5). During the springboard contact phase, total energy and PE increased by $8 \%$ and $29 \%$, respectively, whereas TKE $(-0.3 \%)$ and AKE $(-4 \%)$ remained similar. During the table contact phase, total energy $(-7 \%)$, TKE $(-46 \%)$, and AKE $(-30 \%)$ diminished, whereas PE increased by $27 \%$. During the second flight phase, total energy increased by $9 \%$, attaining a maximal value $10 \%$ higher than that at initial springboard contact. Further, AKE and PE during the second flight phase were $31 \%$ and $15 \%$ higher, respectively, than at push off from the table.

Comparison of Yurchenko and Tsukahara vaults revealed that total energy at initial springboard contact is 7\% lower for Yurchenko vaults than for Tsukahara vaults on average $(p=0.16, \mathrm{r}=0.48)$. However, total energy levels in all other phases of the vault were higher for Yurchenko vaults (mean differences of 4-5\%; $p$ : from 0.12 (landing) to 0.005 (table on); r: from 0.37 (landing) to 0.52 (table on)). Additionally, we found higher TKE during springboard contact and the first flight phase ( $+15 \%$ to $+37 \%$; $p$ : from 0.001 (board on) to 0.0001 (board off and table on); r: from 0.67 (table on) to 0.85 (board on)) but lower PE ( $-3 \%$ to $-17 \%$; $p$ : from 0.25 (board on) to 0.0001 (table on); r: from 0.41 (board on) to 0.84 (table on) ) and AKE ( $-6 \%$ to $-82 \%$; $p$ : from 0.87 (landing) to 0.0001 (board off and table on); r: from 0.02 (landing) to 0.85 (board off and table on)) during all phases for Tsukahara vaults compared to Yurchenko vaults. 


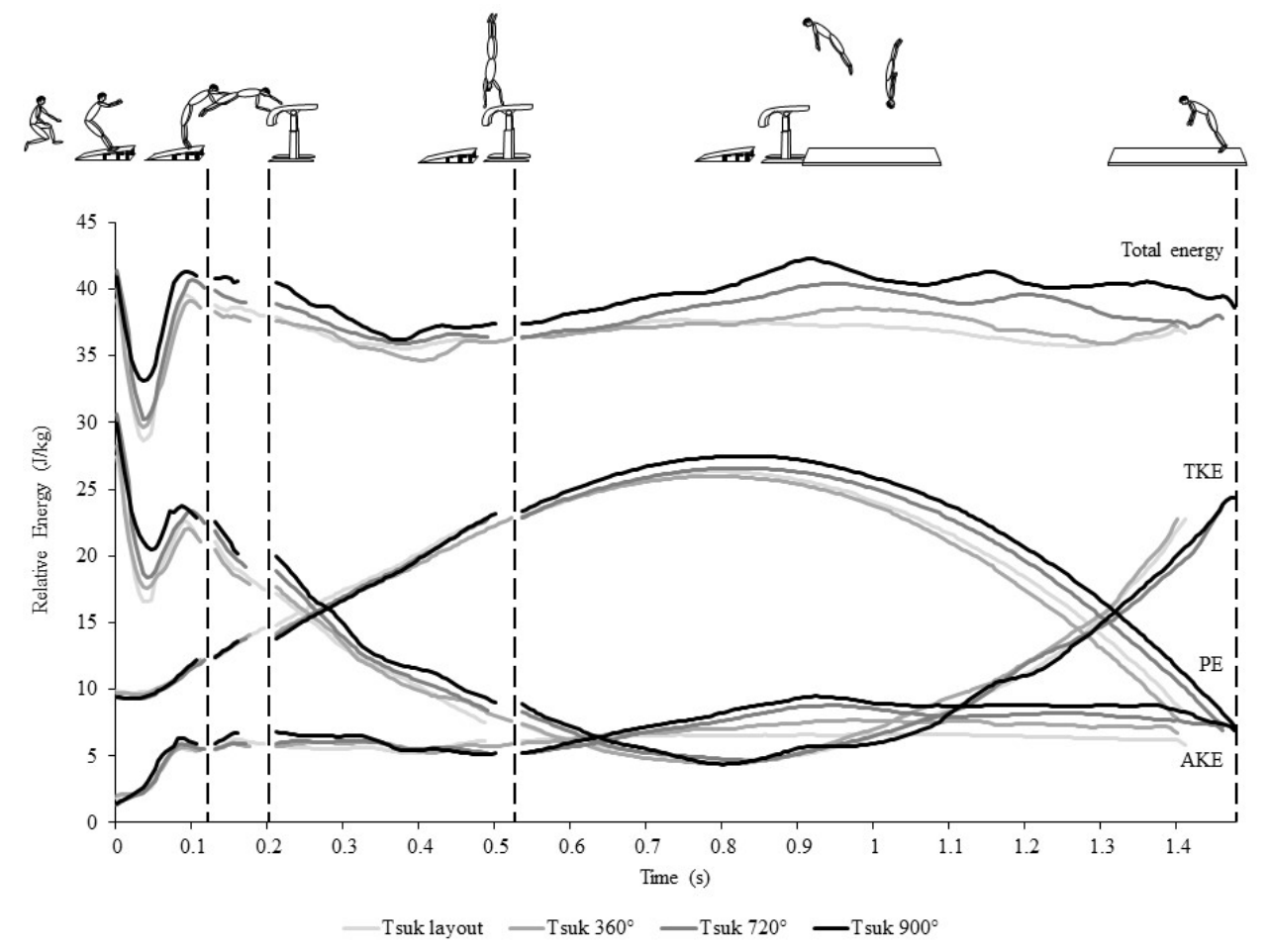

Figure 4. Averaged flux of different energy forms across the different vault phases of simple and difficult Tsukahara vaults (Tsukahara layout without $(n=5)$ and with $360^{\circ}(n=11), 720^{\circ}(n=10)$, and $900^{\circ}(n=2)$ of rotation around longitudinal axis during second flight phase). TKE: translational kinetic energy; PE: potential energy; and AKE: angular kinetic energy.

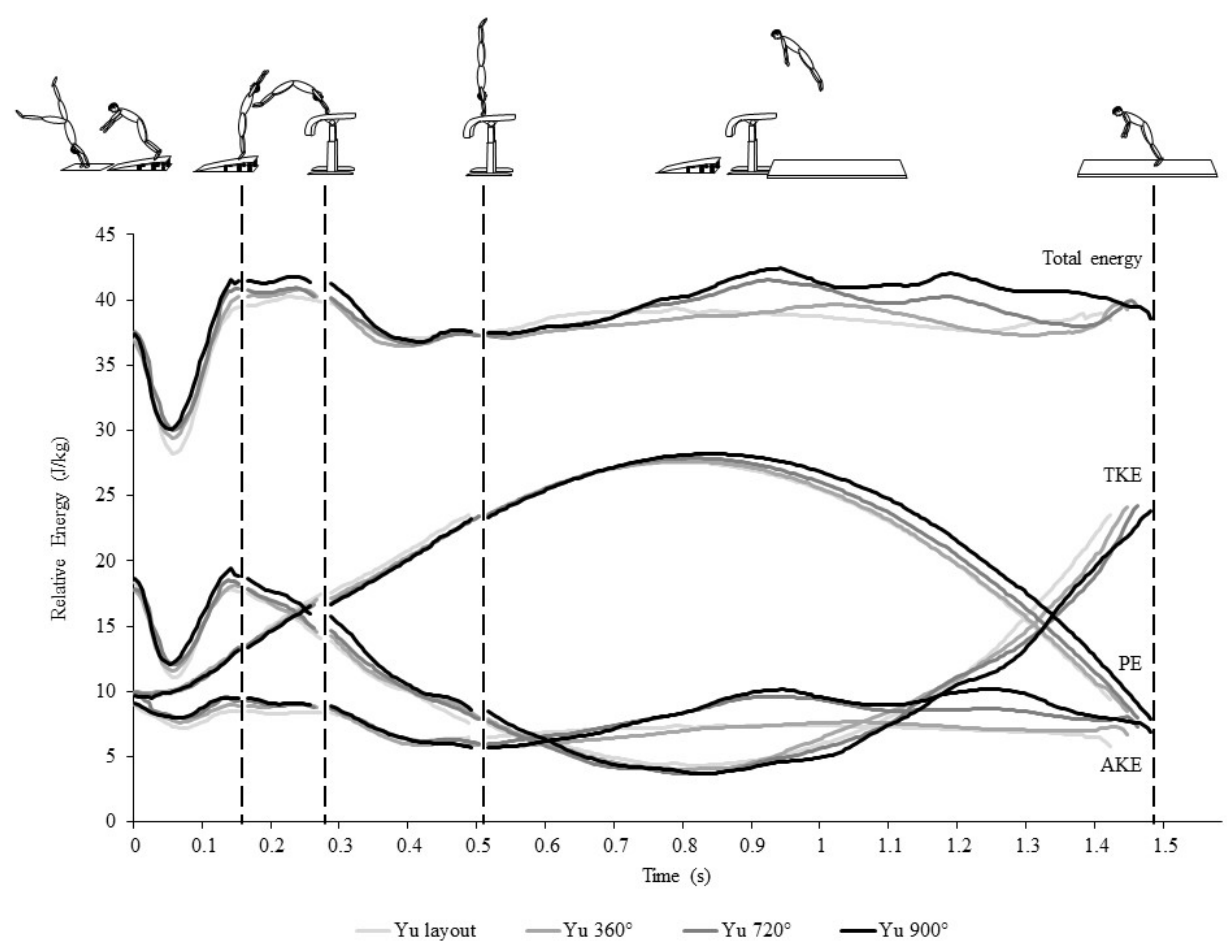

Figure 5. Averaged flux of different energy forms across the different vault phases of simple and difficult Yurchenko vaults (Yurchenko layout without layout $(n=5)$ and with $360^{\circ}(n=4), 720^{\circ}(n=7)$, and $900^{\circ}(n=4)$ of rotation around longitudinal axis during second flight phase). TKE: translational kinetic energy; PE: potential energy; and AKE: angular kinetic energy. 


\subsection{Simple vs. Difficult Yurchenko and Tsukahara Vaults}

Upon initial springboard contact, difficult compared to simple Tsukahara and Yurchenko vaults (layout vs. $\left.900^{\circ}\right)$ were performed with up to $12 \%(p=0.44 ; \mathrm{r}=0.29)$ and $4 \%(p=0.52$; $r=0.20$ ) greater TKE, respectively (Figure 6).
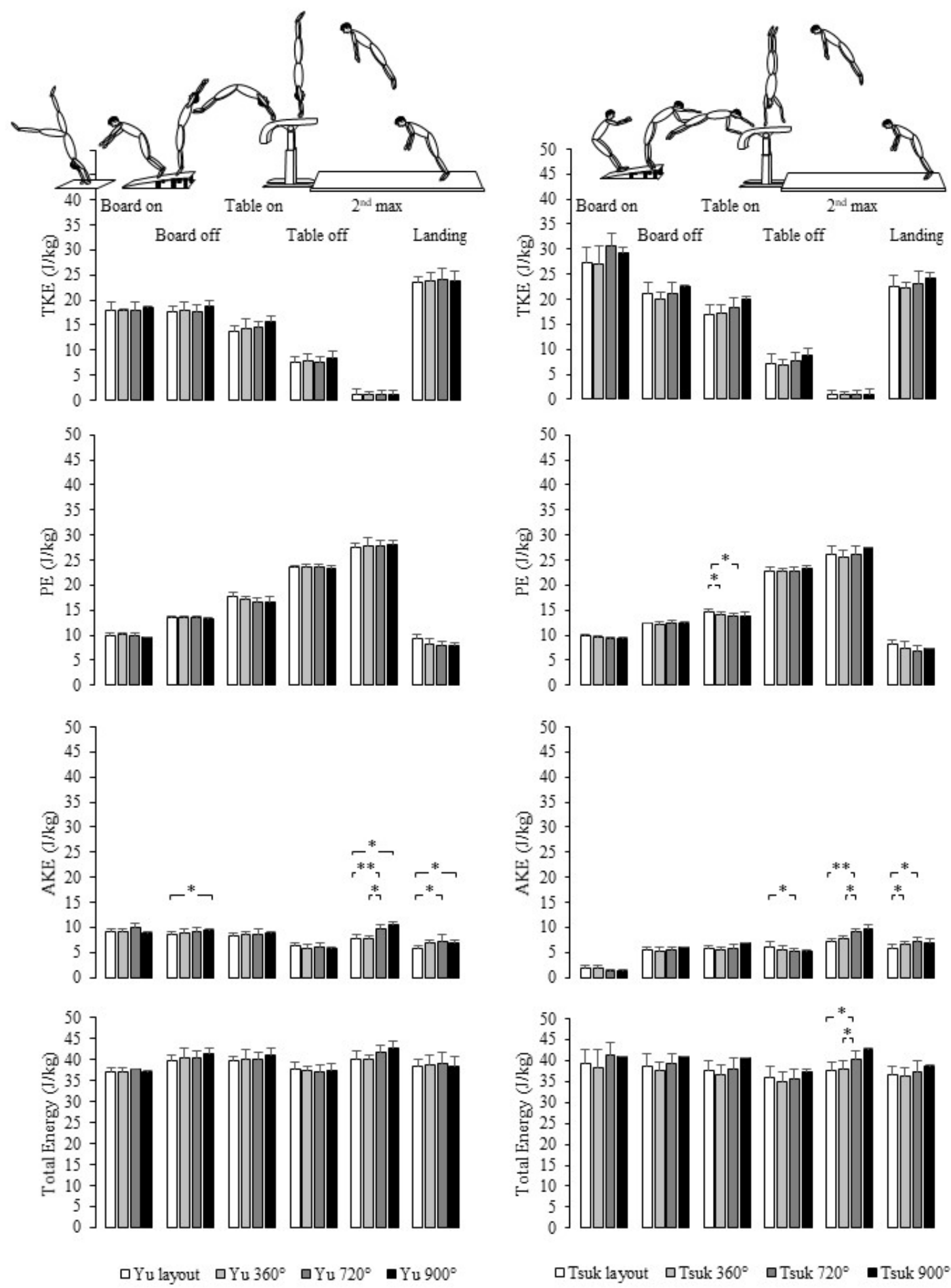

Figure 6. Comparison of various energy forms between simple and difficult Yurchenko and Tsukahara layout vaults without and with $360^{\circ}, 720^{\circ}$, or $900^{\circ}$ of rotation about the longitudinal axis during second flight phase (TKE: Translational kinetic energy; PE: potential energy; AKE: Angular kinetic energy; * $p<0.05 ; * *: p<0.01)$. 
By the end of the springboard contact phase, there was significantly greater AKE for difficult compared to simple Yurchenko vaults $\left(+10 \%\right.$ for layout vs. $\left.900^{\circ} ; \mathrm{r}=0.74\right)$. This difference was somewhat smaller between simple and difficult Tsukahara vaults $(+8 \%$ for layout vs. $900^{\circ} ; p=0.64 ; \mathrm{r}=0.17$ ).

At initial table contact, PE of difficult Tsukahara vaults was significantly lower than for simple Tsukahara vaults ( $-6 \%$ for layout vs. $360^{\circ}, \mathrm{r}=0.52 ;-7 \%$ for layout vs. $900^{\circ}$, $r=0.73)$. A similar (non-significant) difference was observed for Yurchenko vaults $(-6 \%$ for layout vs. $900^{\circ}, p=0.22, \mathrm{r}=41$ ).

At table push off, we found significantly lower AKE for difficult Tsukahara and Yurchenko vaults compared to simple vaults (Tsukahara layout 15\% greater than Tsukahara $900^{\circ}, \mathrm{r}=0.54$; Yurchenko layout $11 \%$ greater than Yurchenko $\left.900^{\circ}, \mathrm{r}=0.65\right)$.

During the second flight phase, AKE was increasingly greater $(+2.4 \%,+21.4 \%$, and $+28.8 \%$ compared to layout) for increasingly difficult Yurchenko vaults $\left(360^{\circ}: p=0.46, \mathrm{r}=0.24 ; 720^{\circ}\right.$ : $\left.\mathrm{r}=0.77 ; 900^{\circ}: \mathrm{r}=0.92\right)$. This was also the case for increasingly difficult Tsukahara vaults $(+9.4 \%$ for $360^{\circ}(p=0.62, \mathrm{r}=0.47),+22.4 \%$ for $720^{\circ}(\mathrm{r}=0.79)$, and $+29.4 \%$ for $900^{\circ}(p=0.05 ; \mathrm{r}=0.73)$, compared to layout). Further, a linear increase in AKE with increasing number of rotations around longitudinal axis during the second flight phase was observed for both vault groups (Spearman's correlation coefficient: Yurchenko: $r=0.83$; Tsukahara: $r=0.99$ ). On average, each additional half rotation $\left(180^{\circ}\right)$ about the longitudinal axis during the second flight phase required between 5.6\% (Tsukahara) and 5.9\% (Yurchenko) more AKE. Total energy during the second flight phase was greater for difficult Tsukahara vaults compared to simple ones $\left(+6.0 \%\right.$ for $720^{\circ}$ compared to $360^{\circ}, \mathrm{r}=0.49 ;+5.2 \%$ for $900^{\circ}$ compared to $720^{\circ}$, $p=0.09, \mathrm{r}=0.49)$; the comparison was repeated for Yurchenko vaults $\left(+4.2 \%\right.$ for $720^{\circ}$ compared to $360^{\circ}: p=0.06, \mathrm{r}=0.57 ;+2.8 \%$ for $900^{\circ}$ compared to $720^{\circ}: p=0.26, \mathrm{r}=0.34$ ). Further, we observed a larger difference of total energy during second flight phase between simple and difficult Tsukahara (layout vs. $900^{\circ}:+12 \%, p=0.53, \mathrm{r}=0.73$ ) compared to simple and difficult Yurchenko vaults (layout vs. $900^{\circ}:+6 \%, p=0.09, \mathrm{r}=0.57$ ).

\section{Discussion}

This study provides new knowledge on the flux of energy by describing and comparing the flux of energy through its various forms during simple and difficult Yurchenko and Tsukahara style vaults performed by male elite gymnasts. This specific topic has only previously been examined for the old vaulting horse [11].

\subsection{Energy Flux of Tsukahara and Yurchenko Vaults}

Our results for Tsukahara vaults show that TKE makes up almost three quarters (72\%) of total energy at initial springboard contact, thus confirming previous investigations and emphasizing importance of a high run-up velocity for performing difficult vaults. For Yurchenko vaults, however, TKE at initial springboard contact is $37 \%$ lower on average, comprising only about half (49\%) of total energy. On the other hand, the characteristic round-off in front of the springboard for Yurchenko vaults generates a substantial amount of AKE (25\% of total energy), which nonetheless enables gymnasts to perform vaults of similar difficulty as with the Tsukahara style. It should be noted, however, that the sufficient AKE is generated only by a technically correct round-off.

For Tsukahara vaults, total energy is more or less preserved $(-2 \%)$ across the springboard contact phase. During this phase however, TKE decreases $(-26 \%)$ as it is mainly transformed into $\mathrm{AKE}(+70 \%)$ and $\mathrm{PE}(+23 \%)$. Consequently, it can be concluded that gymnasts generate most of the AKE necessary to perform Tsukahara vaults during springboard contact. This is contrary to the findings of Krug, Knoll, Köthe, and Zocher [11], who reported a large increase in AKE during the first flight phase.

In contrast to Tsukahara vaults, we found an increase $(+8 \%)$ in total energy during springboard contact for Yurchenko vaults. This can mainly be attributed to an increase in PE $(+27 \%)$, whereas TKE $(-0.3 \%)$ and AKE $(-4 \%)$ remain fairly stable. Our results are in agreement with Penitente et al. [20], who observed a preservation of horizontal 
velocity and an increase in vertical velocity during jump off from the springboard of Yurchenko vaults. Obviously, athletes generate sufficient TKE and AKE with the run-up and round-off in front of the springboard so that translation and rotation must merely be preserved across the springboard contact phase. Hence, the total energy increase during springboard contact may be generated by an effective and powerful jump off. A powerful springboard jump off is realized through a combination of the gymnast's actions and the interaction with the elastic properties of the board and can be better described as a tension-shortening cycle instead of a stretch-shortening cycle. Nevertheless, a jump off from a springboard is closely related to muscle stiffness, and most importantly a high level of reactive strength [20-22], i.e., the ability to generate high ground reaction forces within a short contact time. This relationship emphasizes the importance of physical conditioning for performing difficult vaults.

Interestingly, during table contact, total energy decreases similarly for Tsukahara $(-6 \%)$ and Yurchenko vaults $(-7 \%)$, even though the push off is executed in a completely different position (Yurchenko: two-handed from a backwards handspring; Tsukahara: mainly one-handed sideways from a round off). According to Knoll et al. [23], two-handed push offs (forward handspring) may evoke 2.5 times greater push-off forces than Tsukahara push-offs. Therefore, it seems feasible that either elastic or non-elastic properties of the vaulting table, as well as locomotor properties of the upper body muscles and tendons [24] inhibit a total energy conservation during table contact.

During table contact of Yurchenko vaults, AKE is drastically reduced $(-30 \%)$. The fact that this is achieved with a backwards handspring, and that athletes see the table only shortly before push off, may emphasize the high technical and mental demands of these vaults [3]. Additionally, this corresponds quite expectedly to the intention of the preliminary round-off characterizing this vaulting style, namely, to generate a large amount of AKE, which can subsequently be transformed into PE (height of flight), as was also overserved in the current study. In this context, Knoll [25] observed reductions in angular momentum at push off for Yurchenko vaults between $5 \%$ to $20 \%$.

During table contact of Tsukahara vaults, we found only a slight reduction in AKE $(-6 \%)$. Therefore, height of flight is mostly generated by transforming TKE into PE, whereas the AKE necessary for performing rotational elements during the second flight phase of Tsukahara vaults must be completely generated during jump off from the board. A strong heel strike at the end of the board contact phase in combination with a fast table contact while maintaining the hyperextended body position, may allow the athletes to create the optimal AKE at the jump off from the springboard. During table contact, performing Tsukahara vaults, athletes are required to maintain the AKE despite the conversion of TKE into PE. This can be achieved with a strong push-off from the table with the front support at the end of the table contact phase.

A possible consequence of greater height (and flight time) and more AKE is a better preparation for and execution of the landing, which may benefit the E-score and thus the final score $[3,4,26]$. In this context, we observed greater AKE $(+6 \%)$ and PE $(+5 \%)$ during the second flight phase for Yurchenko vaults than for Tsukahara vaults. This was despite similar gains in total energy $(+9 \%)$ for both vault styles during the second flight phase. This increase in total energy is due in particular to the muscular work, which is necessary to perform the somersaults and twists during the second phase of flight. This can be recognized by the fact that the energy increase is greater when more twists are executed. Consequently, it is important that the athletes perform optimal muscle work for the intended vault during the second flight phase. This can be achieved, for example, by training the twisting technique on the trampoline. Further, total energy during the second flight phase is within $1 \%$ of that at initial springboard contact for Tsukahara vaults, indicating that total energy is merely preserved, while TKE from the run-up is transformed into PE (i.e., height of flight) and rotation during the second flight phase. This may mean that a technically poor execution of take-off and push off from the springboard and table performing Tsukahara vaults can be somewhat compensated by a greater initial TKE (i.e., 
run-up velocity). Nevertheless, the run-up speed cannot be increased at will. The athletes must reach an individually optimal, and therefore controllable, speed, which allows themadapted to their physical conditions in the upper body, as well as their technical abilities when pushing off the table-to effectively convert the energy from the run-up during the board and table contact into height of flight and rotation. In contrast to Tsukahara vaults, total energy during the second flight phase of Yurchenko vaults is $10 \%$ higher than at initial springboard contact, indicating that an important increase in energy is generated (by means of the round-off and back handspring) between run-up and take-off during such vaults. Therefore, proper execution of these pre-take-off elements is crucial for generating the energy necessary for successfully performing difficult Yurchenko vaults.

\subsection{Energy Flux of Tsukahara and Yurchenko Vaults}

At initial springboard contact, the difference in TKE between simple and difficult Tsukahara vaults $(+12 \%)$ is greater than between simple and difficult Yurchenko vaults $(+4 \%)$. This is not surprising and has been reported previously [3-5,7,11]. In order to execute a proper and effective round-off in front of the springboard for Yurchenko vaults, the musculo-tendinal properties of the upper extremities, rather than solely run-up velocity, are a limiting factor [3]. Therefore, run-up velocity must sometimes be modulated to an optimal level due to technical restrictions or poor visual targeting skills in order to hit the board optimally [27].

During springboard contact of Yurchenko vaults, preserving a greater amount of AKE seems to be a crucial factor for increasing vault difficulty (10\% difference between difficult and simple vaults in the current study). This finding is in line with that of Koh and Jennings [28], who emphasized the importance of maintaining a high angular momentum during springboard contact during Yurchenko vaults. For Tsukahara vaults, increasing difficultly requires generating more AKE during springboard contact $(8 \%$ more for difficult compared to simple vaults in the current study). Combined with TKE, this additional AKE represents the exploitable potential to maximize height of flight and rotation during the second flight phase.

The table contact phase of difficult compared to simple Tsukahara and Yurchenko vaults is characterized by up to $7 \%$ lower PE at first table contact a shorter table contact time, and up to $15 \%$ lower AKE at push off. A fast first table contact (low PE) and a short table contact time may be crucial prerequisites for optimally oriented force production, necessary to generate an optimal counter-torque (i.e., reduction in angular momentum) at push off [11], and therefore maximize the height of flight during the second flight phase. Our data seem to confirm that, in order to perform a more difficult vault, a slightly greater counter-torque must be generated. Nevertheless, as previously discussed, the transformation of TKE may be more important for maximizing height of flight than the transformation of AKE into PE. A greater height (and longer duration) of flight increases the time in which to perform rotations around longitudinal and transversal axis (e.g., to perform a more difficult vault). In line with these findings, previous studies observed that it is crucial, in order to perform difficult vaults, that a maximum of horizontal velocity is transformed into vertical velocity during table contact [4,29-32].

Most of the significant differences between simple and difficult vaults in the current study were found for AKE during the second flight phase of Tsukahara and Yurchenko vaults. As all vaults were performed with a layout somersault during the second flight phase, the differences in AKE can be mostly attributed to the different number of rotations around longitudinal axis. In this context, we show that performing $180^{\circ}$ more of rotation around longitudinal axis during the second flight phase requires between $5.6 \%$ and $5.9 \%$ more of AKE. However, AKE accounts for only up to $22 \%$ of the total energy during the second flight phase, whereas two thirds (67\%) of total energy during the second flight phase consists of PE. Therefore, the necessary amount of additional AKE, in order to perform a more difficult vault, is (almost) negligible. Additionally, the requirements for PE and TKE rise slightly with increasing difficulty of the vault. This may lead to the observed 
increase in total energy and to the surprisingly small difference between simple (layout) and difficult $\left(900^{\circ}\right)$ Tsukahara and Yurchenko vaults of $12 \%$ and $6 \%$, respectively. Therefore, coaches and athletes must be aware that, when increasing vault difficulty, regardless of style, demands for several aspects increase slightly but simultaneously. Moreover, it can be said that if an athlete is able to perform a simple vault perfectly, he would theoretically be able to show a more difficult vault (e.g., add a half twist) with a similar amount of energy. In order to be able to show the more difficult vault, therefore, technical and/or mental progress, and not energy-related progress, is particularly necessary.

\section{Conclusions}

In conclusion, we report that, for Yurchenko vaults, a quarter of initial total energy consists of AKE, which is generated with the preliminary round off in front of the springboard. To perform the most difficult Yurchenko vaults, additional energy must be generated during jump off from the springboard, which may depend on physical conditional factors and a technically clean execution of the vault.

With regard to Tsukahara vaults, we conclude that run-up velocity determines the potential to perform difficult vaults almost completely. Even for the most difficult Tsukahara vaults, initial total energy is merely preserved (not increased) during the execution. Hence, an unclean technique may be compensated for, to some degree, with greater run-up velocity.

Finally, we found that in order to perform a more difficult vault $\left(+180^{\circ}\right.$ of rotation around transversal axis) up to $5.9 \%$ of additional AKE is necessary. However, compared to the required PE during the second flight phase, the required additional energy is almost negligible. These findings may help coaches to evaluate each athletes' potential and focus the training on technical and/or physical aspects of the vault performance (Supplementary Materials).

Supplementary Materials: The following are available online at https:/ /www.mdpi.com/article/10 .3390/app11209484/s1, Tables S1-S4: Raw data of translational, potential, angular and total energy of all included vaults.

Author Contributions: Conceptualization, C.S., M.G. and K.H.; methodology, C.S., M.G. and K.H.; formal analysis, C.S., M.G. and K.H.; investigation, C.S., M.G. and K.H.; data curation, C.S., M.G. and K.H.; writing-original draft preparation, C.S.; writing—review and editing, M.G., S.L. and K.H.; supervision, S.L. and K.H.; project administration, C.S. All authors have read and agreed to the published version of the manuscript.

Funding: This research received no external funding.

Institutional Review Board Statement: The study was conducted according to the guidelines of the Declaration of Helsinki, and approved by the Ethics Committee of Bern, Switzerland (Project-ID: 2016-01970, 16 January 2017).

Informed Consent Statement: Informed consent was obtained from all subjects involved in the study.

Data Availability Statement: Data is contained within supplementary material.

Acknowledgments: We would like to thank the athletes and coaches of the Swiss National Artistic Gymnastics Teams for the continuing trust in our scientific projects and for having enabled this study.

Conflicts of Interest: The authors declare no conflict of interest.

\section{Appendix A}

Appendix A.1. Estimate of the Segment's Cylindrical Radius

Segments' radii about the longitudinal axis were calculated by one of the following four methods:

- For femur and humerus segments: the perpendicular distance between the longitudinal axis (i.e., the z-axis provided by PIGP) and the marker on the perimeter of the segment (e.g., 'LTHI', 'LUPA'). 
- For head and pelvis segments: the average perpendicular distance between the longitudinal axis and multiple markers on the perimeter of the segment (i.e., "RASI'/'LASI'/'RPSI' /'LPSI' for the pelvis, and 'RFHD' /'LFHD' /'RBHD' /'RBHD' for the head).

- For tibia and radius (forearm) segments: the mean of the distal and proximal (manually measured) joint widths.

- For hand and foot segments: the (manually measured) proximal joint width

These radii were assumed to be constant (median of all valid frames) and the same for left and right segments (mean of two sides).

\section{Appendix B}

Appendix B.1. Calculating Translational Velocity, Momentum, and Rotational Energy

All translational kinetic and kinematic calculations were based on the trajectory of the whole-body CoM. The three-dimensional velocity vector $(\vec{v})$ expressed the time-normalized frame-to-frame changes in CoM coordinates:

$$
\vec{v}=\left(\frac{d x}{d t}, \frac{d y}{d t}, \frac{d z}{d t}\right)
$$

Scalar velocity $(v)$ was also calculated frame-by-frame as

$$
v=\sqrt{\vec{v}_{x}^{2}+\vec{v}_{y}^{2}+\vec{v}_{z}^{2}}
$$

Translational momentum $(\vec{p})$ was obtained from body mass $\left(m_{b}\right)$ and vector velocity:

$$
\vec{p}=m_{b} \cdot \vec{v}
$$

Translational kinetic energy $\left(E_{t}\right)$ was calculated from body mass and scalar velocity as

$$
E_{t}=\frac{1}{2} \cdot m_{b} \cdot v^{2}
$$

\section{Appendix B.2. Calculating Angular Momentum and Rotational Kinetic Energy}

Remote rotational kinetic energy $\left(E_{r e m}\right)$ was calculated frame-by-frame based on an adaptation of the methods of Kwon, Fortney, and Shin [13] as

$$
E_{\text {rem }}=\frac{1}{2} \cdot m_{k} \cdot\left[\vec{v}_{k}\right]\left[\vec{v}_{k}\right]
$$

where $m_{k}$ is the segment mass, $\vec{v}_{k}$ is the three-dimensional velocity vector of the segment's CoM relative to the body's CoM (first derivative of the three-dimensional positional vector in global coordinates pointing from the body's CoM to the segment's CoM), and $\left[\vec{v}_{k}\right]\left[\vec{v}_{k}\right]$ indicates matrix multiplication.

\section{Appendix B.2.1. Local}

The local angular velocity vector $\left(\vec{\omega}_{\text {loc }}\right)$ of each segment (i.e., about its own CoM) was calculated frame-by-frame using the formula of Wittenburg [14], as cited in Schüler, Schleichardt, Fichtner, and Ueberschär [15], as

$$
\vec{\omega}_{l o c}=2 \cdot \frac{\left(\vec{v}_{1} \times \vec{v}_{2}+\vec{v}_{2} \times \vec{v}_{3}+\vec{v}_{3} \times \vec{v}_{1}\right)}{\left(\vec{v}_{1} \cdot\left(\vec{r}_{2}-\vec{r}_{3}\right)+\vec{v}_{2} \cdot\left(\vec{r}_{3}-\vec{r}_{1}\right)+\vec{v}_{3} \cdot\left(\vec{r}_{1}-\vec{r}_{2}\right)\right)}
$$


where $\vec{r}_{1}, \vec{r}_{2}$, and $\vec{r}_{3}$ are the orthogonal unit vector endpoints corresponding to the segment's local axes, expressed in global coordinates, and $\vec{v}_{1}, \vec{v}_{2}$, and $\vec{v}_{3}$ are the threedimensional velocity vectors (first derivatives) of $\vec{r}_{1}, \vec{r}_{2}$, and $\vec{r}_{3}$, respectively.

The corresponding inertia tensor $\left(\left[J^{\prime}\right]\right)$ for each segment was obtained frame-by-frame by taking the segment's diagonalized inertia tensor $([J])$

$$
J=\left[\begin{array}{ccc}
J_{x x} & 0 & 0 \\
0 & J_{y y} & 0 \\
0 & 0 & J_{z z}
\end{array}\right]
$$

wherein $J_{x x}, J_{y y}$, and $J_{z z}$ are the segment's moments of inertia about its own three principal axes, and transforming it to the global frame using a transformation matrix $(T)$ and the transposition thereof $\left(T^{t}\right)$ with the following equation according to Peraire and Widnall [16]:

$$
\left[J^{\prime}\right]=[T][J][T]^{t}
$$

within $[J], J_{x x}=J_{y y}=m_{s e g} \cdot r_{G}{ }^{2}$ and $J_{z z}=\frac{1}{2} \cdot m_{\text {seg }} \cdot r^{2}$, with $r$ and $r_{G}$ being the segment's radius about the longitudinal axis (cylindrical radius) and its radius of gyration about the other two principal axes, respectively.

Local rotational kinetic energy $\left(E_{l o c}\right)$ was calculated as

$$
E_{l o c}=\frac{1}{2} \cdot\left[J^{\prime}\right]\left[\vec{\omega}_{l o c}\right]\left[\vec{\omega}_{l o c}\right]
$$

Appendix B.2.2. Total

Thereafter, the sum of remote and local angular momenta

$$
\vec{L}_{r e m}+\vec{L}_{l o c}
$$

was further summed for all segments to obtain total angular momentum $(\vec{L})$ :

$$
\vec{L}=\sum_{k}\left(\vec{L}_{r e m}+\vec{L}_{l o c}\right)_{k}
$$

(where $k$ designates a particular body segment), on a frame-by-frame basis. In an analogue manner.

Finally, total rotational kinetic energy $\left(E_{r}\right)$ was calculated as the sum of remote and local rotational kinetic energy, on a frame-by-frame basis, as

$$
E_{r}=\sum_{k}\left(E_{r e m}+E_{l o c}\right)_{k}
$$

(where $k$ designates a particular body segment).

\section{References}

1. FIG. Code of Points Mag (2017-2020); FIG: Lausanne, Switzerland, 2017.

2. Atiković, A.; Smajlović, N. Relation between vault difficulty values and biomechanical parameters in mens artistic gymnastics. Sci. Gymn. J. 2011, 3, 91-105.

3. Schärer, C.; Lehmann, T.; Naundorf, F.; Taube, W.; Hübner, K. The faster, the better? Relationships between run-up speed, the degree of difficulty (d-score), height and length of flight on vault in artistic gymnastics. PLoS ONE 2019, 14, e0213310. [CrossRef] [PubMed]

4. Van der Eb, J.; Filius, M.; Rougoor, G.; Van Niel, C.; De Water, J.; Coolen, B.; De Koning, H. Optimal velocity profiles for vault. In 30th Annual International Symposium on Biomechanics in Sports; Bradshaw, E.J., Burnett, A., Hume, P.A., Eds.; ISBS: Melbourne, Australian, 2012.

5. Fujihara, T. Revisting run-up velocity in gymnastics vaulting. In Proceedings of the 34th International Conference of Biomechanics in Sport, Tsukuba, Japan, 18-22 July 2016; Ae, M., Enomoto, Y., Fujii, N., Takagi, H., Eds.; ISBS: Tsukuba, Korea, 2016 ; pp. 593-596.

6. Brehmer, S.; Naundorf, F. Age related development of run up velocity on vault. Sci. Gymn. J. 2011, 3, $19-27$. 
7. Naundorf, F.; Brehmer, S.; Knoll, K.; Bronst, A.; Wagner, R. Development of the velocity for vault runs in artistic gymnastics for the last decade. In Proceedings of the 26th International Conference on Biomechanics in Sports (2008), Seoul, Korea, 14-18 July 2008; Kwon, Y., Shim, J., Shim, J., Shin, I., Eds.; ISBS: Seoul, Korea, 2008.

8. Schärer, C.; Haller, N.; Taube, W.; Hübner, K. Physical determinants of vault performance and their age-related differences across male junior and elite top-level gymnasts. PLoS ONE 2019, 14, e225975. [CrossRef] [PubMed]

9. Karpowicz, K.; Krych, K.; Karpowicz, M.; Nowak, W.; Gronek, P. The relationship between ca repeat polymorphism of the IGF-1 gene and the structure of motor skills in young athletes. Acta Biochim. Pol. 2018, 65, 43-50. [CrossRef] [PubMed]

10. Prassas, S.; Kwon, Y.H.; Sands, W.A. Biomechanical research in artistic gymnastics: A review. Sports Biomech. 2006, 5, 261-291. [CrossRef] [PubMed]

11. Krug, J.; Knoll, K.; Köthe, T.; Zocher, H.-D. Running approach velocity and energy transformation in difficult vaults in gymnastics. In XVI International Symposium of Biomechanics in Sports; Riehle, H., Vieten, M., Eds.; ISBS: Konstanz, Germany, $1998 ;$ pp. 160-163.

12. Vicon. Vicon Plug-In Gait Product Guide-Foundation Notes Revision; Vicon Motion Systems Ltd.: Oxford, UK, 2010; Volume 2.0.

13. Kwon, Y.-H.; Fortney, V.L.; Shin, I.-S. 3-d analysis of yurchenko vaults performed by female gymnasts during the 1988 seoul olympic games. Int. J. Sports Biomech. 1990, 6, 157-176. [CrossRef]

14. Wittenburg, J. Dynamics of Systems of Rigid Bodies (Volume 33), 2nd ed.; Springer: Berlin/Heidelberg, Germany, 2008.

15. Schüler, A.; Schleichardt, A.; Fichtner, I.; Ueberschär, O. Zur analyse der winkelgeschwindigkeit von rotationsbewegungenvergleich zweier berechnungsmethoden mit beispielen aus dem wasserspringen und weiteren sportarten. In Technologien im Leistungssport 3, Tagungsband zur 19. Frühjahrsschule am 14/15. Mai 2018; Fichtner, I., Ed.; Meyer \& Meyer Sportverlag: Leipzig, Germany, 2018; pp. 89-90.

16. Peraire, J.; Widnall, S. Lecture 126-3d Rigid Body Dynamics: The Inertia Tensor. Available online: https: / /ocw.mit.edu/courses / aeronautics-and-astronautics/16-07-dynamics-fall-2009/lecture-notes/MIT16_07F09_Lec26.pdf (accessed on 1 February 2021).

17. Holm, S. A simple sequentially rejective multiple test procedure. Scand. J. Stat. 1979, 6, 65-70.

18. Fritz, C.; Morris, P.; Richler, J. Effect size estimates: Current use, calculations, and interpretation. J. Exp. Psychol. Gen. 2012, 141, 2-18. [CrossRef] [PubMed]

19. Cohen, J.W. Statistical Power Analysis for the Behavioral Sciences, 2nd ed.; Lawrence Erlbaum Associates: New York, NY, USA, 1988.

20. Penitente, G.; Merni, F.; Fantozzi, S.; Perretta, N. Kinematics of the Springboard Phase in Yurchenko-Style Vaults, 25th ed.; Menzel, H.-J., Chagas, M.H., Eds.; ISBS Symposium: Ouro Preto, Brazil, 2007.

21. Koperski, A.; Kochanowicz, A.; Słodkowski, C. Gymnasts' special quickness-force abilities and the indicators of jump from a springboard. Balt. J. Health Phys. Act. 2010, 2, 139-143. [CrossRef]

22. Motoshima, J.; Kitagawa, J.; Maeda, A. The relationship between the mechanical parameters in the take-off of a vault and the drop jump ability. Sci. Gymn. J. 2015, 3, 37-45.

23. Knoll, K.; Naundorf, F.; Bronst, A.; Wagner, R.; Brehmer, S.; Lehmann, T. 3 Decades of Force Measurement on Vault in Gymnastics, Proceedings of the 32nd International Conference of Biomechanics in Sports, Johnson City, TN, USA, 12-16 July 2014; Sato, K., Sands, W.A., Mizuguchi, S., Eds.; ISBS: Johnson City, WA, USA, 2014.

24. Glasheen, J.W.; McMahon, T.A. Arms are different from legs: Mechanics and energetics of human hand-running. J. Appl. Physiol. 1995, 78, 1280-1287. [CrossRef] [PubMed]

25. Knoll, K. Aufbau und Erhalt des Drehimpulses bei Absprüngen und Abdrücken in technisch-akrobatischen Sportarten. Z. Für Angew. Train. 2004, 11, 72-83.

26. Farana, R.; Vaverka, F. The effect of biomechanical variables on the assessment of vaulting in top-level artistic female gymnasts in world cup competitions. Acta Univ. Palacki. Olomuc. Gymn. 2012, 42, 49-57. [CrossRef]

27. Bradshaw, E.J.; Le Rossignol, P. Anthropometric and biomechanical field measures of floor and vault ability in 8 to 14 year old talent-selected gymnasts. Sports Biomech. 2004, 3, 249-262. [CrossRef] [PubMed]

28. Koh, M.; Jennings, L. Strategies in preflight for an optimal yurchenko layout vault. J. Biomech. 2007, 40, 1256-1261. [CrossRef]

29. Farana, R.; Uchytil, J.; Zahradnik, D.; Jandacka, D. The "akopian" vault performed by elite male gymnasts: Which biomechanical variables are related to a judge's score? Acta Gymnica 2015, 45, 33-40. [CrossRef]

30. Park, C.-H.; Kim, Y.-K.; Joo, J.-Y. Comparative Analysis of Yang Hak-Seon Vault and Tsukahara, Proceedings of the 32nd International Conference of Biomechanics in Sports, Johnson City, TN, USA, 12-16 July 2014; Sato, K., Sands, W.A., Mizuguchi, S., Eds.; ISBS: Johnson City, WA, USA, 2014.

31. Takei, Y. The roche vault performed by elite gymnasts: Somersaulting technique, deterministic model, and judges' scores. J. Appl. Biomech. 2007, 23, 1-11. [CrossRef] [PubMed]

32. Hedbávný, P.; Kalichová, M. Optimization of velocity characteristics of the yurchenko vault. Sci. Gymnast. J. 2015, 7, 37-49. 